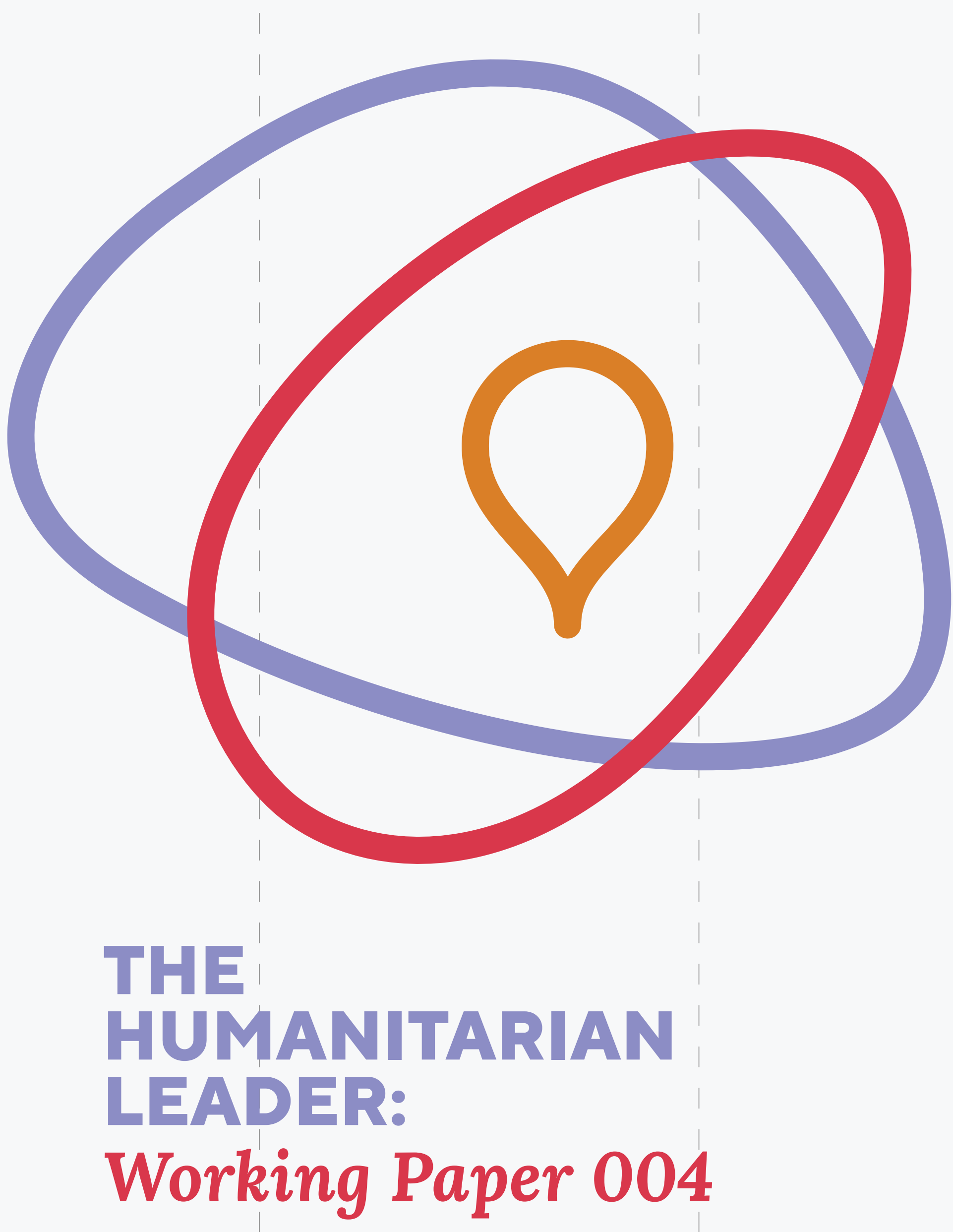




\title{
CHURCH NETWORKS AND LOCALISATION PRACTICE IN THE PACIFIC
}

\author{
Belinda Lauria \\ Aloysius Canete \\ Rebekah Cochrane
}

\section{Working Paper 004}

\section{November 2019}

\section{The Centre For Humanitarian Leadership \\ Deakin University \\ 1 Gheringhap Street \\ Geelong, Victoria 3220}

\section{Belinda Lauria}

Program Manager, Quality and Compliance, International Programs

Anglican Overseas Aid, Australia

blauria@anglicanoverseasaid.org.au

Aloysius Canete

Disaster Management, International Programs

Transform Aid International, Australia

Aloysius.Canete@transformaid.org

Rebekah Cochrane

Projects Officer, International Programs

Transform Aid International, Australia

Rebekah.Cochrane@transformaid.org

This paper was written on behalf of the CAN DO consortium and was prepared for the Centre for Humanitarian Leadership's Working Paper Series.

The views expressed herein are those of the authors and do not necessarily reflect the views of the Centre for Humanitarian Leadership. These papers are circulated for discussion and comment purposes. They have not been peer reviewed.

(C) 2019 by Belinda Lauria, Aloysius Canete and Rebekah Cochrane. All rights reserved. 


\section{ABSTRACT}

The localisation agenda is the largest humanitarian reform in decades. Global research, advocacy and adaptations of localised approaches continue to mature following the World Humanitarian Summit in 2016. The Summit produced The Charter for Faith-based Humanitarian Action, recognising the unique position and comparative advantage of local faith actors (LFAs) in humanitarian settings, owing to their presence in communities before, during, and after crises. More than $80 \%$ of the world's population professes a religious faith, and international development and humanitarian work takes place within communities deeply influenced by faith, with local staff often themselves people of faith (cited in Fletcher 2018, p. 4). LFAs have consistently been among the top implementing partners of UN Agencies in undertaking humanitarian response (UNHCR Partnership Note on faith-based organizations, local faith communities and faith leaders 2014, p.8). Despite this recognition, little has promulgated on the role of LFAs in the localisation agenda and the primacy of LFAs' voices in contextualising the agenda for their communities. Accordingly, CAN DO (Church Agencies Network Disaster Operations) a network of Australian churchbased agencies with established relationships in the Pacific, is building an evidence base to inform international actors and affirm the significance of LFAs in localised humanitarian response within the Pacific region, thereby contributing towards the Charter for Faith-Based Humanitarian Action commitments. This paper is a critical reflection of the 2017-2018 localised response to the Monaro Volcano eruption in Vanuatu. Key learnings frame future collaborations with Pacific churches and pave the road ahead in shifting power differentials, including the advancement of LFAs' role within policy and decision-making at all levels of humanitarian response (Charter for Faith-Based Humanitarian Action 2016, p.2). 


\section{Introduction}

The Church Agencies Network Disaster Operations (CAN DO) is a network of Australian church-based aid and development agencies with long-established relationships in humanitarian and development programs in the Pacific region. Member organisations at present are: Act for Peace, Adventist Development and Relief Agency (ADRA), Anglican Board of Mission (ABM), Anglican Overseas Aid (AOA), Australian Lutheran World Service, Caritas Australia, Transform Aid International (Baptist World Aid Australia), and Uniting World.

CAN DO members are committed to working with local church partners and grassroots organisations to increase their capacity to respond to emergencies, as well as supporting them to build resilience and implement disaster risk reduction activities in their communities. CAN DO is being supported as part of the Australian Humanitarian Partnership (AHP) Disaster Ready Program to build resilience in Papua New Guinea, Fiji, Vanuatu and Solomon Islands. The Disaster Ready Program is being funded by the Australian Government's Department of Foreign Affairs and Trade (DFAT).

\section{Research Purpose}

This paper is the first in a series aimed at introducing CAN DO and its work with churches in the Pacific, in the area of humanitarian localisation. This paper will explore CAN DO's Ambae Volcano Response, which began in Vanuatu in 2017. This response acts as a solid case study for discussing the strengths and challenges associated with implementing a localised, faith-based humanitarian response in the Pacific. It demonstrates the importance of the church's contribution and raises critical questions to be explored moving forward.

DFAT (2019) defines localisation as recognising, respecting and strengthening leadership and decisionmaking by local and national actors in humanitarian action in order to better address the needs of affected populations. This entails:

- Building capacity of national governments, civil society and local communities to prepare for and respond to disasters;

- Working through partner systems informed by appropriate assessments;

- Reinforcing local capacities during a response and ensuring they are not marginalised by international actors arriving with their own staff, systems and priorities;

- Supporting national and local authorities to drive coordination;

- Supporting women as first responders to crises; and

- Supporting better coordinated regional approaches and operational mechanisms.

Churches are often powerful local partners. Pacific churches in particular have high levels of legitimacy and support in their communities and are seen as an essential part of the fabric of their society. Churches are often amongst the first responders in emergencies, drawing on their extensive local resources and understanding of traditional knowledge, leadership and cultures to guide their response. The Charter for FaithBased Humanitarian Action, endorsed during the World Humanitarian Summit in 2016, recognises the unique position and comparative advantage of Local Faith Actors (LFAs) in humanitarian settings, particularly given they are embedded in local contexts before, during, and long after crises occur response (Charter for FaithBased Humanitarian Action 2016, p.2). Indeed, LFAs have consistently been among the top implementing partners of UN Agencies, such as the UNHCR, in delivering humanitarian response. (UNHCR Partnership Note on faith-based organizations, local faith communities and faith leaders 2014, p.8).

Shifting the power to local partners is critical to the success of the localisation agenda. Despite this, there has been little attention given to the role of LFAs in localising humanitarian practice. Constructive partnerships between local churches and other humanitarian actors, including NGOs, will continue to be important to leverage local churches and communities in humanitarian response. This includes recognising the important role that LFAs can play in humanitarian response.

\section{Methodology}

The research undertaken was both quantitative and qualitative. All data analysed was secondary data, as all initial data gathered was undertaken to monitor and report on the humanitarian response to partners and donors.

The rich data gathered throughout the response has now been analysed for this case study and research paper. Data was gathered from the field by local humanitarian actors and other key stakeholders involved in the response. Data was generated from questionnaires, surveys, focus group discussions and key informant interviews, monitoring reports, communication between local and Australian partners, as well as narrative reports and case studies from the local implementing partners. The data captured the voice of the local implementing partners, the two managing CAN DO Australian agencies, affected communities and key stakeholders, including the Vanuatu Police and National Disaster Management Office.

The survey sites were in Maewo and Santo islands, as well as Efate island for the desk-based reports generated by implementing partners. The author analysed and drew findings, achievements and challenges from the data analysis undertaken. 


\section{Situational Overview and Findings}

\section{How CAN DO operationalised}

localisation in this response.

In September 2017 the Manaro Voui Volcano on Ambae Island in Vanuatu erupted. The outpourings of ash and gas damaged infrastructure, destroyed crops and livestock, impacted human health and contaminated water sources. In March 2018, the volcano erupted again and for the second time in six months triggered a state of emergency and the mandatory evacuation of the island's entire population. Many Ambaeans pre-empted the second state of emergency and self-evacuated to the island of Espiritu Santo and as a result, did not receive any official government support.

Humanitarian agencies were initially discouraged from responding on Espiritu Santo, in favour of the official government response centred on Maewo Island. This was despite the majority of displaced people, approximately 8,700, evacuating to Espiritu Santo (cited in AHP Final Report 2018). When the eruption triggered the emergency evacuation, CAN DO immediately became operational via its ongoing and longestablished relationships with local churches in Ambaen communities. The response aimed to strengthen preexisting social support networks, including church leadership, family structures and women's groups that provide stability and social cohesion in times of crisis This was primarily so that communities and families understood the risks and actively prevented children from being exposed to abuse, exploitation, violence, and neglect during displacement and repatriation.

Response activities were designed by ADRA and the Anglican Church of Melanesia (ACOM), in consultation with the National Gender Protection Cluster, in collaboration with ADRA Australia, AOA and the CAN DO Coordination Unit. The response was funded by DFAT through the AHP and managed by ADRA Vanuatu (through ADRA Australia) and ACOM (through AOA).

The expansive Church network enabled localised strategies to begin immediately, with the response locally owned from the outset. The Seventh Day Adventist (SDA) Church (represented by ADRA Vanuatu) and ACOM Vanuatu are established LFAs who share the same worldview with communities and have significant influence on behaviour change and conflict mediation. LFAs have national reach, large established networks and relationships of trust with communities. Almost all of the impacted population of Ambae identify as either Anglican, Church of Christ or Adventist. The Church is a constant within their communities: the Church and their affiliated networks were present before this crisis, (via church core activities and development projects) during the crisis, and will remain present into the future, long after the involvement of most International NonGovernment Organisations (INGOs) has dissipated.

This influence and trust saw two initial Leadership Summits (key activities of the response) reach approximately 3,850 members of the Ambae population
(AHP report, 2018). In addition, ADRA engaged with community members who were originally from Ambae to become members of the Psychosocial First Aid (PFA) teams. This was in order to effectively engage with and build rapport with the affected communities through speaking the local Ambae language and being familiar with local culture and customs.

'Ambae is my home island so, even though the communities were displaced and affected, having someone who knows their religious and cultural background allows them to express themselves openly and willing to receive the trainings'. (ADRA volunteer trainer, 2017).

\section{Collaborative and complementary; roles, resourcing, capacity and relationships}

This paper also explores the difference in staff roles, resourcing, surge capacity and relationships with humanitarian actors between church institutions (ACOM Vanuatu) compared to independent humanitarian agencies owned by a church (ADRA Vanuatu).

CAN DO acknowledges ADRA Vanuatu as a local NGO that is affiliated with an international network of offices. ADRA works collaboratively with local, national and international actors through a complementary process that strengthens local capacity to ensure quality, effective and timely responses to humanitarian crises. Decision making and the country agenda is set nationally, and the international actors genuinely respect and support this model. The role of the international community is to continue to help build local capacity to ensure capability is in place for the national actors to set a strong agenda. This is CAN DO's role with all of its church partners, working towards LFAs being equipped to connect and capably support the national response.

Like all organisations responding to emergencies, the churches have to manage the tension between ongoing responsibilities and critical needs of communities after a disaster. With limited surge capacity and limited private or government funding to respond to emergencies, churches feel significant pressure meeting critical response needs during these times - particularly when responses are protracted.

LFAs also have daily responsibilities that almost exclusively do not involve responding to emergencies. When emergencies do occur and a national response is required, there is the need and expectation for LFAs to divert from their daily responsibilities and contribute to the response. Without surge capacity, this means that daily operations are suspended or neglected (namely their ongoing development projects). Or alternatively, the church is disconnected from the National Disaster Management Office (NDMO) clusters because they are absorbed with their existing day-to-day operations, without time to dedicate to crises as they arise. 
The lack of surge becomes particularly challenging when emergencies are protracted, as in the case of Ambae. When the full and compulsory evacuation was declared through a State of Emergency in mid-2018, and the Government of Vanuatu directed humanitarian assistance for evacuees to Maewo Island only, CAN DO did not have access to the additional financial support required for ACOM's response on Santo. This heavily constrained the Church's assistance capacity and placed enormous pressure on existing human resources.

On the one hand this was a positive for localisation as the Church mobilised primarily through its own means, however it was also a setback as international actors were well-funded, well-connected and commanded a stronger voice in the response coordination. Collectively, further impetus is needed to even out the playing field.

\section{Key Response Activity 1: Leadership Summits}

Four community-led Leadership Summits were held on the islands of Ambae, Maewo and Espiritu Santo. Managed entirely by ACOM and ADRA Vanuatu, the summits allowed LFAs to become architects of their own approach, they organically undertook a strengths-based approach to the design and development of the summits. The summits included representatives from community leadership and established intra-faith collaborations and allowed the sharing of information between different Christian denominations.

The summits mediated partnerships between community leaders, the NDMO and police representatives. Provincial government representatives appreciated the opportunity to meet with and present information to such a large range of community representatives. It was the direct result of these summits that Community Disaster and Climate Change Committees (CDCCCs) were reactivated and Ward Councils formed to work with their Area Council Administrator in all aspects of disaster preparedness and response, along with the establishment of a Church Task Force. Representatives from the summits returned to their communities with plans for community engagement specific to locally identified needs in disaster risk reduction, food security and protection of women and children. The second Leadership Summit focused on peacebuilding and was held to address the cultural tensions and conflict between relocated communities from Ambae and host communities of Maewo. Facilitators used theological teachings as an entry point for LFAs to critically examine the differentiations between biblical and cultural concepts of protection and inclusion, reflecting on adapted cultural traditions that may inhibit protection.

Community leaders from both relocated and host communities were able to move from strangers to a more cohesive community. Practical actions to foster peace building and reconciliation were recorded between the evacuees from Ambae and the host communities of Maewo to uphold peace. ACOM reported that all participants have concluded to bring about reconciliation and peace.
'Today I can see some light. I have been walking in darkness on how to properly cultivate my land but now I know. I now have clear direction for tomorrow with my Ambae families; I have plans to allocate plots of land to individual families. So, thank you very much indeed for these much needed tools. God bless'. (Maewo Chief, Interview, 2017)

'This way forward has been brought back to their [community leader's] respective camps for implementation to enable peace building. We believe very strongly that whenever their way forward is in its implementation process, both parties will live in peace and harmony and will no longer see each other as strangers and aliens but citizens'. (ACOM staff member, internal report, 2017).

\section{Key Response Activity 2: Participatory Evaluation Planning and Design}

The mid-term redesign and evaluation planning saw a focus on complementary capacity building. CAN DO provided technical assistance and ACOM led the workshops and design to ensure the process itself reflected localisation. Local collaborators were involved in the design at the outset and participated in decisionmaking as equals. Activities elicited participatory reflection, program improvement and organisational development. Evaluation planning through the workshop has been assessed as transformative, building capacity to sustain change - in both the participating organisations and in the sector more broadly, confirming the high importance in the strength and alignment of participatory evaluation planning with localisation in humanitarian response. Major results included indicators for measuring localisation in this response.

'Collaborative workshops have significantly improved the technical capacity of M\&E (monitoring and evaluation) practices for Vanuatu implementing agencies. The localisation workshop and practice in evaluation techniques have both contributed to this'. (ACOM staff member, internal report 2017).

'Your continuous technical, moral and spiritual support has enabled a successful response which is unique because it's not just a one-sided response but people are holistically fed: physically, mentally and spiritually'. (ACOM staff member, internal report 2017).

\section{Key Response Activity 3: \\ Distribution of dignity kits, hygiene and protection awareness}

ADRA Vanuatu volunteers implemented a dual-pronged approach in their initial response efforts. Volunteers met immediate health needs via the distribution of dignity kits while also creating a soft entry point to build women's awareness and knowledge in identifying gender-based violence (GBV) throughout the distribution phase. The contents of the kits and distribution locations were decided upon by feedback 
from volunteers in consultation with the Gender and Protection Cluster. Volunteers shared information on where women could access GBV services, including the counselling centre and local police stations, using the national referral pathway developed by the Gender and Protection cluster. As women and girls collected their kits, they were provided with the opportunity to share and voice their challenges and concerns, including health and safety issues. Volunteers actively engaged with the women and girls, organising community awareness-raising sessions, educational one-on-one talks and group counselling.

Community women expressed their gratitude for the delivery of dignity kits, noting that shop owners had closed or stopped ordering sanitary goods. A group of teenage girls also said that their usual means of affording sanitary products, being earnings gained for gardening crops, was no longer feasible after the disaster. Women reported using cut baby nappies for sanitary purposes, however with water access shortages, women could only wash the cut nappies in the sea and hang them in full view of men and boys, who could see their "rubbish" as one woman said.

'Girls and women really need the items that ADRA are now giving us through these dignity kits to maintain our health and hygiene...Thank you so much for providing these kits to us in this community because you gave us some hope to continue'. (Ambaen woman, interview, 2017)

\section{Key Response Activities 4 \& 5: Safeguarding Initiatives, ADRA Vanuatu and World Vision Vanuatu}

As a disaster preparedness and resilience activity, ADRA Vanuatu had been training volunteers using a survivor-centred approach in the prevention of sexual violence and exploitation and the protection of the vulnerable over the past two years. During the Ambae response, institutions and systems for physical and social protection weakened as relevant officers and their families were also affected. Volunteers worked closely with the West Ambae Committee Against Violence Against Women (CAVAWs, established by the Vanuatu Women's Centre). There were further breakdowns in community support systems and protection mechanisms when children were left on Espiritu Santo to attend school whilst their parents returned to Ambae.

Volunteers divided into teams and worked with communities to develop community plans to reach all of West Ambae. The plans were comprised of gender and awareness campaigns, mental health and psychological support, Psychosocial First Aid activities, and child safeguarding awareness. Volunteers also conducted three Psychosocial First Aid and Psychosocial Service trainings with 80 church and youth leaders. As volunteers have been provided with Family Life and GBV awareness by qualified family counsellors, they were able to guide teams to assess needs and concerns, particularly of women, girls, teachers and chiefs. Needs that were prioritised included water, sanitation and hygiene (WASH) and food security. Critical information was passed onto the Gender and Protection Cluster, who then referred on to the Food Security and WASH Clusters.

'I initially did not see the importance of providing Psychological First Aid support to people in disasters, but going through the training, I now can make connection which I think it is really important in times of disaster'. (Ambae response actor, interview, 2017).

Theology was an effective entry point to build capacity of local church partners and Christian communities, elevating social inclusion and equality by finding alignments between humanitarian principles, namely GBV, child protection and religious values. Similarly, World Vision's Channels of Hope program is an evidenced-based approach which aligns theological messages to societal issues, changing attitudes and behaviours towards women and family violence. Due to the success of the project in Melanesia, CAN DO commissioned World Vision Vanuatu (WVV) to facilitate this program during the response.

WVV's training methodology, approach, and delivery subscribes to the localised mandate, with training content contextualised to address needs identified by affected communities and delivered to community leaders by ni-Vanuatu facilitators.

The effect of this approach was captured in testimonials from participants:

'I have never heard any teachings about child protection; I would have taught my children and their spouses to be more careful with safety over their children, as well as understanding the importance of child protection, especially in times of crisis'. (Maewo female leader, interview, 2017)

When interviewed in 2017, a community priest expressed the training's transformative effect:

'Training has recuperated my strength through a spiritual wake up call. I will work with colleagues on return to our campsite to put together a plan to do awareness activities and teachings, because together, we can build a strong and resilient community. (Community priest, interview, 2017)

World Vision Vanuatu's Channels of Hope Evaluation report (2017) states that upon returning to their communities, all participants from the Santo-based training spread key messages through a variety of mediums. They had become champions and community trainers.

Training was focused on addressing the needs communicated by field staff and working with affected communities, however the poor psychological state of the participants was underestimated. Facilitators 
from WVV were initially faced with difficulties in delivering the training on Maewo due to the trauma that participants were experiencing. The training placed participants in a vulnerable position by asking them to reflect and share recent, raw, traumatic experiences. This raises many questions: Does the accountability for this oversight fall with local field staff to identify and predict this consequence? Is the onus on CAN DO to provide stronger opportunities for skill development of field staff? Or alternatively, as the contractor of WVV, did CAN DO unintentionally control the power dynamic, limiting the local partner's freedom to express concerns about the training's approach?

Facilitators identified that a higher number of chiefs should have been included in the training as this would have worked towards mediating the three main systems of civil society: the Church, the chiefly system, and government. While there was documentation of transformative behaviours without ongoing mentoring or refresher training, it is not known if community leaders will continue with the initial momentum participants gained to mobilise communities through advocacy efforts. CAN DO may need to rethink the approach to short-term capacity development, instead facilitating ongoing opportunities for LFAs to practice skills and continue to build capacities on an ongoing basis. Albeit, such a shift would start with sector wide conversations and negotiations, namely with donors; as capacity building with large numbers of beneficiaries is challenging, with funding constraints and short-term implementation timeframes. This is an example where the need for greater synergy between humanitarian response activities and longer-term development projects such as Pacific Disaster Ready, proves vital.

Churches have made significant gains in areas of gender violence, and because of this, we feel confident to begin addressing awareness of LGBTQI rights with the same approach. (AHP report, 2017).

\section{Challenges to localising response coordination mechanisms}

ACOM Vanuatu and local faith leaders reported feeling disconnected from the country-level decision-making process during the response. The perception articulated by many of these leaders, and in fact some ni-Vanuatu representatives from the clusters, was that the humanitarian response was being heavily influenced by international humanitarian actors. Other international participants told of the need for local actors, including churches, to not only participate in the clusters, but to have a genuine and empowered voice that represented the interests of their constituents.

This experience is not only related to the Pacific. The Active Learning Network for Accountability and Performance in Humanitarian Action (2018) provided a longitudinal assessment of the humanitarian system. Global trends indicate a general lack of coordination between agencies, poor technical competencies by actors and traditional international interventions failing to be effective but continuing to be used.

The report suggests a need for humanitarian work to be built on practise, grounded in evidence and reflected upon in learning forums. CAN DO has taken this reflective and collaborative approach by continuing to engage across faith and secular organisations to strengthen the work of the Church and LFAs. CAN DO is also taking an auxiliary approach to localisation, supporting the Church and LFAs to complement and strengthen national disaster management operations.

In contrast to ACOM's experience, ADRA is a regular member of the cluster system and as such all activities, including the timing and implementation of activities, was undertaken in consultation with the Gender and Protection Cluster. ADRA was well connected with the clusters and other stakeholders responding to the crisis, working with the Department of Women's Affairs, the co-lead (Department of Youth and Sport) and with IsraAid. ADRA had well-coordinated implementation of its activities with the Cluster Lead, the Cluster Co-Lead, IsraAid and the Vanuatu Humanitarian Team. Upon later reflection, it became evident that ACOM and ADRA had some differing experiences and challenges during the response, with communication systems and channels between the two local implementing organisations as well as their respective Australian NGO partners an opportunity for improvement in the future.

'We are not clear on how the Church should be providing support because we are not connected to the cluster system put in place by the government'. (ACOM staff member, interview 2017).

\section{The need for greater support and surge capacity}

ACOM and AOA field trips to relocation sites during the early recovery period highlighted the failure of the official response to take advantage of the existing strengths of the Church. Consultations focussed on traditional leadership while failing to engage with church leadership. There were also reports that ADRA and ACOM could strengthen their communication, relationships and collaboration with other churches. The need to embark on coalition building and collaboration to increase the collective voice of the church at the national level and within the cluster system became evident through the regional Church coordination unit, Vanuatu Council of Churches.

As such, it is critical that other humanitarian actors, including CAN DO, support the churches' efforts to build their resilience, coordination effectiveness and surge capacity, while acknowledging their ongoing traditional church work in communities. For example, with the support of CAN DO members ABM and AOA, ACOM has appointed a local disaster coordinator within the Church. The most effective means of developing a support network around this role is being explored. In contrast, ADRA, a standalone relief and development 
agency, affiliated and owned by the SDA Church, has a designated Emergency Response Coordinator who works with ADRA and the SDA Church to coordinate effective and efficient humanitarian responses. Questions around shifting the power and navigating the difficult landscape of a truly equitable partnership between international and local actors must be reflectively acknowledged and addressed.

This response aimed for LFAs to be better prepared and more resilient as first responders. The foundations have been set to improve collaboration and communication between community leaders and government officials for further homogenous, coordinated humanitarian responses, with ongoing communication and engagement needed by LFAs. Further engagement and relationship building is needed to enhance mediations within and between the churches themselves, as well as with the chiefly system and government to improve dialogue and advocate for enhanced coordination in humanitarian responses.

'We would like to humbly apologise for breakdown of communication with VCC resulting in nonparticipation during this [the final Leadership Summit] response. The general observation during this response delivery was that there is still a lot to be done by churches in upholding peace, and the welfare of the Ambaeans as well as preparing for any future disaster. There is great value of collaboration in humanitarian response, and recognition of the value of intermediary organisations providing technical, financial, and advocacy support to local partners'. (ACOM staff member, interview 2017)

\section{Safeguarding challenges}

CAN DO observes recognised global good practice for beneficiaries and staff, acting within a mandate of inclusion and do no harm. This differs from some adaptations of customary practices that cause harm to women and children in the Pacific.

During the Ambae response, CAN DO's theological approach and safeguarding entry points addressed this challenge by connecting spiritual wellbeing to overall wellbeing for communities; invoking faith as a strength that advocates for equality and justice for all. While there was evidence of short-term transformative behaviours after protection training and support from church partners, longer-term transformation will need sustained and widely adopted behaviour change, which will require many diverse messages that are ongoing and interactive.

Advocates for localisation call for shifting the power from international organisations to local actors as a necessary, long overdue action that the humanitarian system needs to embrace. And yet, one of the largest challenges facing the localisation transformation is observing global safeguarding standards in contexts where they are not strictly observed by duty bearers, as well as the challenge of mainstreaming human rights within a non-paternalistic framework.

Some academics in women's empowerment and gender in development, pursue the idea that ethical transformative change through external interventions that elevate gender justice can be facilitated. One eminent researcher, Kabeer (2019), explains that external agents can open possibilities and spaces for women, but external interventions and agencies must ensure they do not push their own agenda. International actors can provide a space that women otherwise would not have been provided, where women can then begin to question social constructs.

Kabeer (2019) advocates for an autonomous and selfgoverning space to be provided for women by external interventions. This school of thought has earlier roots, including Bourdieu's (1997) concept of Doxa regarding cultural norms. Bourdieu (1997) explains that what is taken for granted and appears as the natural order within a community, such as oppressive cultural norms that do not change over time, is due to the lack of choices or possibilities presented or made available to that community.

Decreasing the risk of abuse and exploitation of females caused by adapted customary traditions was addressed during the Ambae response. According to Bourdieu (1997) such socially accepted injustices can only be questioned (including by women and girls themselves) when a new view is presented that provides choice and enables individuals to question their world order. A similar approach was applied by CAN DO through protection training, local faith actors and theologians who used the Bible as the vehicle to advocate for the elevation of safeguarding, protection and human rights. Communities were provided with a different interpretation of biblical teachings that have been unquestionably accepted for generations, namely, messages that promoted and sustained harmful societal constraints on women and girls.

CAN DO is currently undertaking and documenting a safeguarding review to strengthen the inter-agencies' safeguarding processes and culture between its Australian member agencies. It will then work with Pacific partners, accompanying and complementing their work to socialise enhanced safeguarding practices. LFAs' voices will lead and guide the process for their communities. CAN DO members and Church partners will collaboratively reflect on institutional approaches that have struggled to generate change, and emerging approaches that are leading the way to meet the rights and needs of the vulnerable.

While the sentiment of shifting the power is not new, and ongoing research and evaluations are directed towards localised approaches, the international community is yet to practice all that they promote. 
According to the Humanitarian Advisory Group (HAG) (2019) there is a disparity between international actors' perception of their transition towards a more localised approach compared to the perception of their efforts from local actors. Not surprisingly, international actors perceive their work as more advanced, effective and impactful compared to local actors' perceptions, who reported international agencies' progress at a much less advanced stage.

HAG (2019) reports there is some evidence of momentum from international actors to provide more decision-making power to national and local actors. Unfortunately, there was only limited evidence of actual activity or impact to evidence this shift, and the majority of 'localised approaches' were not systemic, but rather, informal and with limited scope.

\section{Conclusion}

Reflecting on the lessons from the Ambae disaster response, key questions have emerged that CAN DO will seek to explore into the future. Each of these will continue to frame the work of CAN DO with Pacific churches, to help the network deliver more effective, localised responses.

This paper has proposed that when working with Pacific churches, the humanitarian community needs to take a strengths-based approach. As such, the CAN DO consortium is a good example of promoting localisation in the Pacific, where church partners are collaborating and utilising their longstanding presence in the community to deliver effective coordination and response.

In the case of Ambae, the CAN DO church partner response was driven by those who had a long-term stake in affected areas. Working through churches ensured the response addressed the self-identified, holistic needs of people affected by the emergency. 


\section{REFERENCES}

Anglican Overseas Aid and the Adventist Development and Relief Agency 2018, Australian Humanitarian Partnership Activation Final Report, Unpublished Ambae humanitarian response report for the Australian Government's Department of Foreign Affairs and Trade, AOA and ADRA, Melbourne and Sydney.

Deer, C. (2008) "Doxa," in Grenfell, M. (ed.) Pierre Bourdieu: Key Concepts. Acumen Publishing, pp. 119-130. doi: 10.1017/UPO9781844654031.011.

Department of Foreign Affairs and Trade 2019, 'Localisation of humanitarian Assistance' presentation notes/paper presented at the Localisation Experts Dialogue, Melbourne, 31 January.

Fletcher, $\mathrm{G}$ on behalf of the Australian Council for International Development (ACFID) Sexual Rights in Development Community of Practice, 2018 'Faith, sexual orientation, gender identity and the ACFID Code of Conduct', pp. 7-9.

Kabeer, N 2019, ' The Conditions and Consequences of Choice, Reflections on the Measurement' United Nations Research Institute for Social Development Discussion Paper No. 108, retrieved March 2019, <http://www.unrisd.org/80256B3C005BCCF9/ (httpAuxPages)/31EEF181BEC398A380256B67005B720A /\$file/dp108.pdf>.

The Agenda for Humanities, 2016, Charter for FaithBased Humanitarian Action, retrieved January 2019, $<$ https://www.agendaforhumanity.org/ initiatives/4012>

The Humanitarian Advisory Group 2019, LOCALISATION IN VANUATU: DEMONSTRATING CHANGE, report is part of the Humanitarian Advisory Group's Intention to impact: Localisation of humanitarian action in the Pacific research project, retrieved January 2019,

<https://humanitarianadvisorygroup.org/wp-content/ uploads/2019/02/Vanuatu-Baseline-Report_

February-2019_FINAL.pdf>

United Nations High Commission for Refugees, 2014, Partnership Note on faith-based organizations, local faith communities and faith leaders, retrieved 10 January 2019 < https://www.unhcr.org/539ef28b9.pdf>

World Vision Vanuatu 2018, World Vision Vanuatu's Channels of Hope Completion Report, Unpublished Ambae Volcano Emergency Response Program report, WVV, Luganville Vanuatu. 